\title{
EQUILIBRIA AND THEIR STABILITY FOR A VISCOUS DROPLET MODEL
}

\author{
PATRICK GUIDOTTI
}

\begin{abstract}
A classical model of fluid dynamics is considered which describes the shape evolution of a viscous liquid droplet on a homogeneous substrate. All equilibria are characterized and their stability is analyzed by a geometric reduction argument.
\end{abstract}

\section{INTRODUCTION}

Consider the shape of a droplet of a viscous liquid on a homogeneous substrate and denote by $\Omega(t)$ the region wetted by the liquid. The droplet can then be described by a height field $u: \Omega(t) \rightarrow \mathbb{R}$ at any given time during the evolution (at least in the regime of interest). Asymptotic and averaging techniques yield, on appropriate assumptions (very small and very viscous droplet, see [5]), a simplification of Navier-Stokes equations which is considered here. The system in question reads

$$
\begin{cases}-\Delta u=\lambda & \text { in } \Omega(t), \text { for } t>0, \\ u=0 & \text { on } \partial \Omega(t), \text { for } t>0, \\ \int_{\Omega(t)} u d x=V_{0}>0 & \text { for } t>0, \\ V=F(|\nabla u|) & \text { on } \partial \Omega(t), \text { for } t>0, \\ \Omega(0)=\Omega_{0} . & \end{cases}
$$

The nonlinearity $F$ drives the evolution, i.e. the contact angle dynamics via the fourth equation in (1.1) for the front velocity $V$ in outward normal direction $\nu(t)$ to the surface $\Gamma(t)=\partial \Omega(t)$. It is easily seen that balls evolve invariantly (maintaining their shape) for these equations and that a radius $r_{e}$ is singled out by the the volume conservation constraint combined with any canonical choice of $F$ such as

$$
F(s)=s^{2}-1 \text { or } F(s)=s^{3}-1, s>0,
$$

and that such equilibrium ball is stable for the corresponding ordinary differential equation it satisfies (cf. [5). For the purposes of this paper it will only be assumed that

$$
F^{\prime}>0 \text { and that } F(1)=0 \text {, }
$$

thus effectively prescribing the contact angle below which the droplet would tend to locally retract and above which it would locally expand. These are the minimal qualitative assumptions of any physically relevant model.

It will be shown here that circles are the only equilibria of (1.1) and that they are stable with respect to any smooth perturbation. In particular, a perturbed circle will converge exponentially fast back to a circle albeit centered at a possibly different point. Its radius is, however, uniquely determined by volume conservation and therefore remains unchanged as compared to that of the circle being perturbed. The proof of stability hinges on the explicit computation of the linearization of (1.1) in a circle and on the use of a special nonlinear coordinate system in the "space of curves" suggested by the translation invariance of the system.

Previous results about this basic model of fluid dynamics include local and global existence of appropriate weak solutions [6, 7] which would cover instances where singularity formation can

Key words and phrases. Droplet model, contact line evolution, stationary solutions, stability. 
occur (see the numerical experiments of 4]) and a local well-posedness result [2] in the category of classical solutions.

The remainder of the paper is organized as follows. In the next section the results of [2] are briefly summarized in order to introduce the appropriate functional setup and since they form the starting point for the subsequent analysis. Then equilibria are characterized and their stability is investigated by a fully explicit calculation of the linearization of the nonlinear, nonlocal curve evolution described by the last two equations of (1.1) and obtained by thinking of the other unknown $u$ as the function of $\Gamma(t)$ determined by solving the first three equations, combined with the introduction of convenient nonlinear coordinates in the "space of curves" $\Gamma$ with respect of which the linearization in the equilibrium circle coincides with the computed one. In these nonlinear coordinates the evolution admits a simplified description of the dynamics obtained by a direct and revealing exploitation of the translation invariance of the system. The proposed approach has to be contrasted with the more complicated and widespread approach typically taken to deal with similar problems sharing some geometric invariance (translation invariance in the specific case) with (1.1). Indeed center manifold analyses [3] or a generalized principle of stability [9] were previously used. Both, albeit to a different degree, are more involved than the direct and transparent approach suggested in this paper.

\section{Setup}

In order to reduce system (1.1) to an evolution equation for a simple unknown, an appropriate parametrization of $\Gamma(t)$ is necessary. To that end, fix a $\mathrm{C}^{\infty}$-hypersurface $\Gamma$ close to $\Gamma_{0}=\partial \Gamma_{0}$ in such a way that the latter can be described as a graph in normal direction over $\Gamma$, i.e.

$$
\Gamma_{0}=\left\{x+\rho_{0}(x) \nu(x) \mid x \in \Gamma\right\},
$$

for some function $\rho_{0}: \Gamma \rightarrow \mathbb{R}$. For technical reasons (better invariance properties with respect to interpolation) so-called little Hölder spaces prove a convenient choice of phase space. It is recalled that, for $\alpha \in(0,1)$ and an open subset of $\mathbb{R}^{n}$, the space of bounded uniformly Hölder continuous functions of exponent $\alpha$

$$
\operatorname{BUC}^{\alpha}(O)=\left\{u: O \rightarrow \mathbb{R} \mid\|u\|_{\infty}<\infty,[u]_{\alpha}:=\sup _{x \neq y} \frac{\mid u(x)-u(y)}{|x-y|^{\alpha}}<\infty\right\}
$$

is a Banach space with respect to the norm

$$
\|\cdot\|_{\alpha, \infty}=\|\cdot\|_{\infty}+[\cdot]_{\alpha} .
$$

For $k \in \mathbb{N}$ one also defines

$$
\mathrm{BUC}^{k+\alpha}(O)=\left\{u \in \mathrm{BUC}^{k}(O)\left|\partial^{\beta} u \in \mathrm{BUC}^{\alpha}(O) \forall\right| \beta \mid=k\right\},
$$

which is a Banach space with respect to the norm $\|\cdot\|_{k, \alpha}=\|\cdot\|_{k, \infty}+\sup _{|\beta|=k}\left[\partial^{\beta} \cdot\right]_{\alpha}$. Here it is used that

$$
\mathrm{BUC}^{k}(O)=\{u: O \rightarrow \mathbb{R} \mid u \text { is } k \text {-times continuously differentiable }\}
$$

and $\|\cdot\|_{k, \infty}=\sup _{|\beta| \leq k}\left\|\partial^{\beta} u\right\|_{\infty}$. The the little Hölder spaces are given by

$$
h^{k+\alpha}(O)=\text { closure }_{\|\cdot\|_{k, \alpha}}\left(r_{O} \mathcal{S}\left(\mathbb{R}^{n}\right)\right),
$$

i.e. as the completion of the restriction of smooth rapidly decreasing functions to the set $O$ with respect to the $\mathrm{BUC}^{k+\alpha}$ topology. These spaces can all be transplanted on any smooth compact manifold by the use of standard localization techniques and a smooth partition of unity. This is how the notation $h^{k+\alpha}(\Gamma)$ should be interpreted. Any given function $\rho \in h^{2+\alpha}(\Gamma)$ yields a 
diffeomorphism $\theta_{\rho}$ between $\Gamma$ and $\Gamma_{\rho}=\{x+\rho(x) \nu(x) \mid x \in \Gamma\}$, which can be extended to a diffeomorphism of $\mathbb{R}^{n}$ still denoted by $\theta_{\rho}$ such that

$$
\theta_{\rho}: \mathbb{R}^{n} \rightarrow \mathbb{R}^{n}, y \mapsto \begin{cases}X(y)+[\Lambda(y)+\varphi(\Lambda(y)) \rho(X(y))] \nu(X(y)), & y \in \Omega_{\Lambda}, \\ y, & y \notin \Omega_{\Lambda},\end{cases}
$$

where $\varphi$ is a smooth cut-off function, $\Omega_{\Lambda}$ is a tubular neighborhood of $\Gamma$ and $(X(y), \Lambda(y))$ are "tubular coordinates" of $y$, i.e. they satisfy

$$
y=X(y)+\Lambda(y) \nu(x(y))
$$

Then

$$
\theta_{\rho}(\Omega)=\Omega_{\rho}, \theta_{\rho}(\Gamma)=\Gamma_{\rho},\left.\theta_{\rho}\right|_{\Omega_{\Lambda}^{c}}=\mathrm{id} .
$$

Clearly the tubular neighborhood is taken as small as the geometry of $\Gamma$ requires in order to obtain a well-defined coordinate system $(X(y), \Lambda(y))$ and $\rho$ small enough as to ensure that $\Gamma_{\rho} \subset \Omega_{\Lambda}$. More explicit and quantitative assumptions can be found in 2] but are not needed in the remainder of this paper. It should, however, be observed that the smallness assumption on $\rho \in h^{2+\alpha}(\Gamma)$ is immaterial since $\Gamma \in \mathrm{C}^{\infty}$ can be chosen arbitrarily close to $\Gamma_{0}$ so that $\rho_{0}$ will be small. For the purpose of the analysis to follow this represents no restriction. With the diffeomorphism $\theta_{\rho}$ in hand, the first three equations of (1.1) can be pulled back to a fixed domain $\Omega$ to give

$$
\begin{cases}\mathbb{A}(\rho)=-\theta_{\rho}^{*} \Delta \theta_{*}^{\rho} u=\lambda & \text { in } \Omega, \text { for } t>0 \\ u=0 & \text { on } \Gamma, \text { for } t>0 \\ \int_{\Omega} u\left|D \theta_{\rho}\right| d x=V_{0}>0 & \text { for } t>0\end{cases}
$$

by means of the pull-back

$$
\theta_{\rho}^{*}: h^{2+\alpha}\left(\Gamma_{\rho}\right) \rightarrow h^{2+\alpha}(\Gamma), u \mapsto v=u \circ \theta_{\rho},
$$

and the associated push-forward $\theta_{*}^{\rho}$ given by its inverse. This can be done on the assumption that $\Omega(t)=\Omega_{\rho(t, \cdot)}$ for a given

$$
\rho:[0, T] \rightarrow h^{2+\alpha}(\Gamma),
$$

yielding $\Gamma(t)=\Gamma_{\rho(t, \cdot)}$ and satisfying

$$
\rho(t, \cdot) \in \mathbb{B}_{h^{1+\alpha}(\Gamma)}(0, \delta) \cap h^{2+\alpha}(\Gamma)=: \mathcal{V} \text { for } t \in[0, T],
$$

for a small $\delta>0$ as described in 2]. It is useful to denote the solution of (2.1) obtained by fixing $\rho \in \mathcal{V}$ and setting $\lambda=1$ by

$$
\theta_{\rho}^{*} u_{\rho}=v_{\rho}=\mathbb{S}(\rho) \mathbf{1},
$$

where $\mathbb{S}(\rho)=\lambda(\rho) \overline{\mathbb{S}}(\rho)$ for $\theta_{\rho}^{*} \bar{u}_{\rho}=\bar{v}_{\rho}=\overline{\mathbb{S}}(\rho) \mathbf{1}$ which solves

$$
\left\{\begin{array} { l l } 
{ \mathbb { A } ( \rho ) v = 1 } & { \text { in } \Omega \text { for } t > 0 , } \\
{ v = 0 } & { \text { on } \Gamma \text { for } t > 0 , }
\end{array} \text { or, equivalently, } \left\{\begin{array}{ll}
-\Delta u=1 & \text { in } \Omega_{\rho} \text { for } t>0, \\
u=0 & \text { on } \Gamma_{\rho} \text { for } t>0,
\end{array}\right.\right.
$$

in the original coordinates, and

$$
\lambda(\rho)=\frac{V_{0}}{\int_{\Omega} \bar{v}_{\rho}\left|D \theta_{\rho}\right| d y}=\frac{V_{0}}{\int_{\Omega_{\rho}} \bar{u} d x},
$$

rescales the solution to satisfy the volume constraint. As shown in [2, it is observed in passing that operators or other quantities depending on $\rho$, do indeed depend analytically on it. This fact will be needed later. In order to reformulate the kinematic equation in (1.1), it is convenient to define

$$
N_{\rho}: \Omega_{\Lambda} \rightarrow \mathbb{R}, y \rightarrow \Lambda(y)-\rho(X(y)),
$$


so that

$$
\Gamma_{\rho}=N_{\rho}^{-1}(0) \text { and } \nu_{\rho}(y)=\frac{\nabla N_{\rho}(y)}{\left|\nabla N_{\rho}(y)\right|}, y \in \Gamma_{\rho} .
$$

Then it can be written

$$
V(y, t)=\frac{\rho_{t}}{\left|\nabla N_{\rho}(y)\right|}=F\left(\left|D u_{\rho}\right|\right) .
$$

Observing that $|D u|=-\partial_{\nu} u$ on $\Gamma_{\rho}$ in this particular case, the following single scalar nonlinear, nonlocal evolution equation for the "shape function" $\rho$ results

$$
\begin{cases}\rho_{t}=\left|\nabla N_{\rho}\right| F\left(-\partial_{\nu_{\rho}} u_{\rho}\right)=\left|\nabla N_{\rho}\right| F\left(-\lambda(\rho) \partial_{\nu_{\rho}} \bar{u}_{\rho}\right)=: G(\rho) & \text { on } \Gamma \text { for } t>0, \\ \rho(0)=\rho_{0} & \text { on } \Gamma .\end{cases}
$$

It follows from 22 that (2.2) is equivalent to the original problem in the context of classical solutions, that $G$ depends analytically on $\rho$ and that

Theorem 2.1. Given $\rho_{0} \in \mathcal{V}$, there exists $T>0$ and a unique solution

$$
\rho \in \mathrm{C}^{1}\left([0, T], h^{1+\alpha}(\Gamma)\right) \cap \mathrm{C}\left([0, T], h^{2+\alpha}(\Gamma)\right)
$$

of (2.2) and, thus, a solution $\left(\mathbb{S}(\rho) \mathbf{1}, \Omega_{\rho}\right)$ of (1.1).

The proof relies on localization, perturbation, and optimal regularity results for parabolic equations which make it possible to reduce local well-posedness to properties of the linearization $D G\left(\rho_{0}\right)$ in the initial datum. The abstract approach of [2] is necessary to deal with the most general case but does not provide any explicit representation for the linearization. It is qualitative in nature and only yields that $D G\left(\rho_{0}\right)$ is the infinitesimal generator of an analytic semigroup on $h^{1+\alpha}(\Gamma)$ with domain $h^{2+\alpha}(\Gamma)$. Here and in order to obtain the stability results outlined earlier a much more detailed understanding of $D G\left(\rho_{0}\right)$ is necessary in the special case when $\Gamma=\Gamma_{0}=\mathbb{S}_{r_{e}}$.

\section{Equilibria}

While the results remain valid for any space dimension, the analysis would have to be adapted to the specific dimension considered. The technique would essentially coincide in all dimensions but the specific spherical functions involved would have to be chosen depending on the dimension. In order to avoid rendering the presentation unnecessarily cumbersome, the choice is made to consider the case $n=2$ in this paper.

An equilibrium solution of (1.1) is obtained if $\left(u_{e}, \Omega_{e}\right)$ can be found such that

$$
V=F\left(\left|D u_{e}\right|\right)=0 .
$$

On the assumptions made earlier this is the case only if

$$
\partial_{\nu} u_{e}=-\left|D u_{e}\right| \equiv 1 \text { on } \Gamma_{0},
$$

so that $u_{e}$ satisfies

$$
\begin{cases}-\Delta u_{e}=\lambda, & \text { in } \Omega \\ u_{e}=0, & \text { on } \Gamma \\ \partial_{\nu} u_{e}=-1, & \text { on } \Gamma_{0}\end{cases}
$$

A classical rigidity result by Serrin [11] then implies that a classical solution of the above overdetermined system can only exist if $\Omega_{e}$ is a circle of some radius $r_{e}$ determined by the additional requirement that

$$
\int_{\Omega_{e}} u_{e} d x=V_{0}
$$


Theorem 3.1. If $\left(u_{e}, \Omega_{e}\right)$ is a steady-state of (1.1), then $\Omega_{e}$ must be a circle $\mathbb{S}_{r_{e}}$ of radius

$$
r_{e}=\sqrt[3]{\frac{4 V_{0}}{\pi}} \text { and } u_{e}=\frac{1}{2}\left(r_{e}-\frac{r^{2}}{r_{e}}\right)
$$

where $r$ is the distance from the center of the circle. The parameter $\lambda$ satisfies $\lambda=2 / r_{e}$.

Proof. Serrin's classical result implies that $\Omega_{e}$ is a sphere. The rest follows from a direct computation.

Remarks 3.2. (a) The author of [5] include a partial stability result. They fix a center of the circle and derive and ode describing the evolution of a circle of initial radius $r_{0}$ with the same center. They show that the circle of radius $r_{e}$ is locally asymptotically stable.

(b) In order to obtain a more general stability result the center and, more, in general, the geometry needs to be perturbed as well. The "freedom in the choice of center" is responsible for the existence of a (translational) eigenvalue $0 \in \sigma\left(D G\left(\rho_{e}\right)\right.$ where $\rho_{e} \equiv 0$ when the reference manifold is the stationary solution $\mathbb{S}_{r_{e}}$ itself.

(c) The functions in the kernel of $D G(0)$ can be computed by parametrizing the shifted circle

$$
\Omega_{\epsilon v}=\mathbb{S}_{r_{e}}+\epsilon v,
$$

over the stationary reference circle which can be assumed to be centered in the origin without loss of generality. To do so, a function $h_{\epsilon}: \Omega \rightarrow \mathbb{R}$ needs to be determined such that

$$
\left(r_{e}+h_{\epsilon}\right)\left[\begin{array}{l}
\cos (\theta) \\
\sin (\theta)
\end{array}\right]-\epsilon\left[\begin{array}{l}
v_{1} \\
v_{2}
\end{array}\right]=r_{e}\left[\begin{array}{c}
\cos (\phi) \\
\sin (\phi)
\end{array}\right] .
$$

This yields

$$
h_{\epsilon}(\theta)=-r_{e}+\sqrt{r_{e}^{2}+\epsilon^{2}|v|^{2}+2 \epsilon r_{e} v \cdot \nu_{e}}
$$

where

$$
\nu_{e}=\left[\begin{array}{c}
\cos (\theta) \\
\sin (\theta)
\end{array}\right], \tau_{e}=\left[\begin{array}{c}
-\sin (\theta) \\
\cos (\theta)
\end{array}\right]
$$

can be thought of as the unit outward normal and unit tangent to the circle $\mathbb{S}_{r_{e}}$, respectively. The angle $\theta$ satisfies

$$
\tan (\theta)=\frac{r_{e} \sin (\phi)+\epsilon v_{2}}{r_{e} \cos (\phi)+\epsilon v_{1}}, \arctan (\theta)=\frac{r_{e} \cos (\phi)+\epsilon v_{1}}{r_{e} \sin (\phi)+\epsilon v_{2}},
$$

and, for $\epsilon<<1$, is uniformly close to $\phi$, i.e.

$$
\theta_{\epsilon}\left(v_{1}, v_{2}\right)(\phi) \rightarrow \phi \text { as } \epsilon \rightarrow 0
$$

uniformly in $v \in \mathbb{S}_{1}$. It follows that

$$
\left.\frac{d}{d \epsilon}\right|_{\epsilon=0} h_{\epsilon}\left(\theta_{\epsilon}\right)=\frac{1}{2 r_{e}} 2 r_{e} \nu_{e} \cdot v=v_{1} \cos (\theta)+v_{2} \sin (\theta)
$$

is an element of the kernel of $D G(0)$ for any $\mathbb{R}^{2}$.

(d) It will be shown that the kernel only consists of the above "translational" eigenvectors. Translation also yields a manifold of equilibria $\mathcal{E}$ (the set of all circle with fixed radius $r_{e}$ ) which can locally be parametrized as in (c). It in fact corresponds to a global center manifold for the evolution. The analysis which follows does, however, not make use of any abstract results about center manifolds but rather exploits directly the translation invariance. 


\section{LiNEARIZATION}

Consider now the equilibrium $\mathbb{S}_{r_{e}}$ centered at the origin and use it as the reference manifold $\Gamma$ so that the equilibrium solution $\rho_{e}$ vanishes identically. Then

$$
X(y)=r_{e} \frac{y}{|y|}, \Lambda(y)=|y|-r_{e}, \nu_{e}=\frac{y}{|y|},
$$

and $y=X(y)+\Lambda(y) \frac{y}{|y|}$. In this case the function $N_{\rho}$ is simply given by

$$
N_{\rho}(y)=|y|-r_{e}-\rho(y /|y|) .
$$

For ease of computation and notation the Euclidean coordinate $y$ or the polar $(r, \phi)$ will be used interchangeably. In particular, functions on $\mathbb{S}_{r_{e}}$ will be identified with functions of the angle variable $\phi$. With this convention one has

$$
\nabla N_{\rho}=1 \frac{\partial}{\partial r}-\rho^{\prime}(\phi) \frac{\partial}{\partial \phi} \text { and }\left|\nabla N_{\rho}\right|^{2}=\left(r_{e}+\rho\right)^{2}+\rho^{\prime}(\phi)^{2} .
$$

It can easily be seen that

$$
\left.\frac{d}{d \epsilon}\right|_{\epsilon=0} \frac{\left|\nabla N_{\epsilon h}\right|}{r_{e}+\epsilon h}=0, h \in h^{2+\alpha}(\Gamma) .
$$

When computing the linearization it is therefore possible to replace any occurrence of $\frac{\left|\nabla N_{\epsilon}\right|}{r_{e}+\rho}$ by its value in $\epsilon=0$ and thus consider only $F\left(-\lambda(\epsilon h) \partial_{\nu_{\epsilon h}} \bar{u}_{\epsilon h}\right)$ which leads to

$$
\left.\frac{d}{d \epsilon}\right|_{\epsilon=0} G(\epsilon h)=-\left.F^{\prime}(1) \frac{d}{d \epsilon}\right|_{\epsilon=0}\left[\lambda(\epsilon h) \partial_{\nu_{\epsilon h}} \bar{u}_{\epsilon h}\right]
$$

It turns out, contrary to the approach taken in [2, that it is more convenient not to perform the transformation to a fixed domain when computing the linearization in a circle.

Theorem 4.1. Let $h \in h^{2+\alpha}(\Gamma)$. Then

$$
\left.\frac{d}{d \epsilon}\right|_{\epsilon=0} \partial_{\nu_{\epsilon h}} \bar{u}_{\epsilon h}=\frac{4 V_{0}}{\pi r_{e}^{4}}\left[r_{e} D T N_{\mathbb{S}_{r_{e}}}(h)-h\right],
$$

where $D T N_{\partial \Omega}$ is the so-called Dirichlet-to-Neumann operator, i.e. the operator mapping a Dirichlet datum $h$ to the outward normal derivative $\partial_{\nu} w_{h}$ of the solution $w_{h}$ of the boundary value problem

$$
\begin{cases}-\Delta w=0, & \text { in } \Omega, \\ w=h, & \text { on } \partial \Omega .\end{cases}
$$

Remark 4.2. The above theorem provides a formula for the first "domain" variation of the solution of

$$
\begin{cases}-\Delta u=1, & \text { in } \Omega, \\ u=0, & \text { on } \partial \Omega,\end{cases}
$$

in the circle of radius $r_{e}$, i.e. $\left.\frac{\partial}{\partial \Omega} u\right|_{\Omega=\mathbb{S}_{r_{e}}}$.

Proof. Consider the solution $\bar{u}_{\epsilon h}$ of (4.2) for $\Omega=\Omega_{\epsilon h}$ and look for it in the form

$$
\bar{u}_{\epsilon h}=w_{\epsilon h}+\frac{1}{2}\left(r_{e}^{2}-|x|^{2}\right) \text {. }
$$

Then clearly

$$
-\Delta \bar{u}_{\epsilon h}=-\Delta w_{\epsilon h}+1=1 \text { or }-\Delta w_{\epsilon h}=0,
$$

and

$$
\left.\bar{u}_{\epsilon h}\right|_{\Gamma_{\epsilon h}}=\left.w_{\epsilon h}\right|_{\Gamma_{\epsilon h}}+\frac{1}{2}\left(r_{e}^{2}-\left(r_{e}+\epsilon h\right)^{2}\right) \text { or }\left.w_{\epsilon h}\right|_{\Gamma_{\epsilon h}}=\epsilon r_{e} h+\frac{\epsilon^{2}}{2} h^{2} .
$$


It follows that

$$
\partial_{\nu_{\epsilon h}} \bar{u}_{\epsilon h}=\partial_{\nu_{\epsilon h}} w_{\epsilon h}+\partial_{\nu_{\epsilon h}} \frac{1}{2}\left(r_{e}^{2}-|x|^{2}\right)=r_{e} D T N_{\Omega_{\epsilon h}}\left(\epsilon h+\frac{\epsilon^{2}}{2 r_{e}} h^{2}\right)-\left(r_{e}+\epsilon h\right) \nu_{\epsilon h} \cdot \nu_{e} .
$$

Since $\nu_{\epsilon h}=\left(-\epsilon h^{\prime} \tau_{e}+\left(r_{e}+\epsilon h\right) \nu_{e}\right) / \sqrt{\left(r_{e}+\epsilon h\right)^{2}+\epsilon^{2} h^{\prime 2}}$ one has that

$$
\partial_{\nu_{\epsilon h}} \bar{u}_{\epsilon h}=r_{e} D T N_{\Omega_{\epsilon h}}\left(\epsilon h+\frac{\epsilon^{2}}{2 r_{e}} h^{2}\right)-\frac{\left(r_{e}+\epsilon h\right)^{2}}{\left[\left(r_{e}+\epsilon h\right)^{2}+\epsilon^{2} h^{\prime 2}\right]^{1 / 2}} .
$$

Now, if $D T N_{\Omega_{\rho}}$ depends continuously on $\rho$, it can be easily inferred that

$$
\left.\frac{d}{d \epsilon}\right|_{\epsilon=0} \partial_{\nu_{\epsilon h}} \bar{u}_{\epsilon h}=r_{e} D T N_{\mathbb{S}_{r_{e}}}(h)-h .
$$

The continuous dependence, however, follows from

$$
\operatorname{DTN}_{\Omega_{\rho}}(h)=\partial_{\nu_{\rho}} \bar{u}_{\rho}=\left.\frac{\nabla N_{\rho}}{\left|\nabla N_{\rho}\right|} \cdot \nabla \bar{u}_{\rho}\right|_{\Gamma_{\rho}},
$$

for

$$
\bar{u}_{\rho}=-\left(\theta_{\rho}^{*} \Delta_{\mathbb{S}_{r_{e}}} \theta_{*}^{\rho}, \gamma_{\Gamma_{\rho}}\right)^{-1}(1,0)
$$

for $\rho \in \mathcal{V}$. It can be seen as in $[2]$ that

$$
\left[\rho \mapsto D T N_{\Omega_{\rho}}\right]: h^{2+\alpha}\left(\Gamma_{\rho}\right) \rightarrow \mathcal{L}\left(h^{2+\alpha}\left(\Gamma_{\rho}\right), h^{1+\alpha}\left(\Gamma_{\rho}\right)\right)
$$

is an analytic function because $N_{\rho}$ and $\theta_{\rho}$ depend algebraically on $\rho$. Notice that $h: \mathbb{S}_{r_{e}} \rightarrow \mathbb{R}$, that is, a function of the angle variable $\phi$ only, can always be transplanted on $\Gamma_{\rho}$ to and identified with the function $\tilde{h}: \Gamma_{\rho} \rightarrow \mathbb{R}$ via

$$
\tilde{h}\left(\left[\left(r_{e}+\rho(\theta)\right] \nu_{e}(\theta)\right)=h(\theta), \theta \in[0,2 \pi) .\right.
$$

Thus the operator $D T N_{\Omega_{\rho}}$ can be viewed as defined on the fixed space $h^{2+\alpha}\left(\mathbb{S}_{r_{e}}\right) \widehat{=} h_{p}^{2+\alpha}$, where the latter is the space of $2 \pi$-periodic little Hölder functions.

In order to complete the evaluation of the linearization, the term

$$
\left.\frac{d}{d \epsilon}\right|_{\epsilon=0} \lambda(\epsilon)=\left.\frac{d}{d \epsilon}\right|_{\epsilon=0} \frac{V_{0}}{\int_{\Omega_{\epsilon h}} u_{\epsilon h} d x} .
$$

needs to be evaluated. Thus consider

$$
\left.\frac{d}{d \epsilon}\right|_{\epsilon=0} \int_{\Omega_{\epsilon h}} u_{e}(x) d x=\int_{\Gamma_{\epsilon h}} u_{e}(x) d \sigma_{\Gamma_{\epsilon h}}(x)=0,
$$

by the boundary condition, so that

$$
\left.\frac{d}{d \epsilon}\right|_{\epsilon=0} \int_{\Omega_{\epsilon h}} u_{\epsilon h}(x) d x=\left.\int_{\mathbb{B}\left(0, r_{e}\right)} \frac{d}{d \epsilon}\right|_{\epsilon=0} u_{\epsilon h}(x) d x .
$$

Using a Green's function representation for the solution, i.e.

$$
u_{\epsilon h}(x)=\int_{\Omega_{\epsilon h}} G_{\epsilon h}(x, \bar{x}) d \bar{x},
$$

this amounts to computing

$$
\left.\frac{d}{d \epsilon}\right|_{\epsilon=0} u_{\epsilon h}=\int_{\mathbb{S}_{r_{e}}} G_{0}(x, \bar{x}) d \sigma_{\mathbb{S}_{r_{e}}}(\bar{x})+\left.\int_{\mathbb{B}\left(0, r_{e}\right)} \frac{d}{d \epsilon}\right|_{\epsilon=0} G_{\epsilon h}(x, \bar{x}) d \bar{x}=\left.\int_{\mathbb{B}\left(0, r_{e}\right)} \frac{d}{d \epsilon}\right|_{\epsilon=0} G_{\epsilon h}(x, \bar{x}) d \bar{x} .
$$

Notice that the boundary integral term vanishes because the Green's function for the Dirichlet problem is zero on the boundary. For the last term it is resorted to the so-called Hadamard 
domain variation formula (see [10] for a generalized version and more recent developments) for Green's functions which, in this particular case, yields

$$
G_{\epsilon h}(x, \bar{x})-G_{e}(x, \bar{x})=\epsilon \int_{0}^{2 \pi} \partial_{r} G_{e}\left(x, r_{e}, \phi\right) \partial_{r} G_{e}\left(\bar{x}, r_{e}, \phi\right) h(\phi) d \phi+o(\epsilon),
$$

for any $h \in \mathrm{C}^{2+\alpha}\left(\mathbb{S}_{r_{e}}\right)$. In order to continue the computation it is convenient to have an explicit formula for the Dirichlet Green's function for the circle of radius $r_{e}$

$$
G(r, \theta, \bar{r}, \bar{\theta})=\frac{1}{4 \pi} \log \left[\frac{r_{e}^{2} r^{2}+r_{e}^{2} \bar{r}^{2}-2 r_{e}^{2} r \bar{r} \cos (\theta-\bar{\theta})}{r^{2} \bar{r}^{2}+r_{e}^{4}-2 r_{e}^{2} r \bar{r} \cos (\theta-\bar{\theta}}\right]
$$

from which it follows that

$$
\partial_{r} G\left(r, \theta, r_{e}, \phi\right)=\frac{r_{e}^{2}}{2 \pi} \frac{r_{e}^{2}-r^{2}}{r_{e}^{2} r^{2}+r_{e}^{4}-2 r_{e}^{3} r \cos (\theta-\bar{\theta})}=\frac{1}{2 \pi} \frac{r_{e}^{2}-r^{2}}{r^{2}+r_{e}^{2}-2 r_{e} r \cos (\theta-\bar{\theta})} .
$$

It is important to observe that that the function

$$
(r, \theta) \mapsto \int_{0}^{2 \pi} \partial_{r} G\left(r, \theta, r_{e}, \phi\right) g(\phi) d \phi
$$

is harmonic in $\mathbb{B}\left(0, r_{e}\right)$ with boundary value $g$ on $\mathbb{S}_{r_{e}}$. Combining everything it can see that

$$
\begin{gathered}
\left.\int_{\mathbb{B}\left(0, r_{e}\right)} \int_{\mathbb{S}_{r_{e}}} \frac{d}{d \epsilon}\right|_{\epsilon=0} G_{\epsilon h}(x, \bar{x}) d \bar{x} \\
=\int_{0}^{2 \pi} \int_{0}^{r_{e}} \int_{0}^{2 \pi} \frac{1}{2 \pi} \frac{r_{e}^{2}-r^{2}}{r^{2}+r_{e}^{2}-2 r_{e} r \cos (\theta-\phi)} \frac{1}{2 \pi} \frac{r_{e}^{2}-\bar{r}^{2}}{\bar{r}^{2}+r_{e}^{2}-2 r_{e} \bar{r} \cos (\bar{\theta}-\phi)} h(\phi) d \phi \bar{r} d \bar{r} d \bar{\theta} \\
=\int_{\mathbb{B}\left(0, r_{e}\right)} \int_{0}^{2 \pi} \frac{1}{2 \pi} \frac{r_{e}^{2}-r^{2}}{r^{2}+r_{e}^{2}-2 r_{e} r \cos (\theta-\phi)} \frac{r_{e}^{2}}{2} d \phi r d r d \theta \\
=\frac{r_{e}^{2}}{2} \int_{0}^{2 \pi} h(\phi) \int_{0}^{2 \pi} \int_{0}^{r_{e}} \frac{1}{2 \pi} \frac{r_{e}^{2}-r^{2}}{r^{2}+r_{e}^{2}-2 r_{e} r \cos (\theta-\phi)} r d r d \theta d \phi=\frac{r_{e}^{4}}{4} \int_{0}^{2 \pi} h(\phi) d \phi=\frac{\pi r_{e}^{4}}{2} \hat{h}_{0},
\end{gathered}
$$

where $\hat{h}_{0}$ is the average of the function $h$. Returning to the computation of the linearization it is seen that

$$
\left.\frac{d}{d \epsilon}\right|_{\epsilon=0} \frac{V_{0}}{\int_{\Omega_{\epsilon h}} u_{\epsilon h} d x}=-\left.\frac{V_{0}}{\left(\int_{\Omega_{\epsilon h}} u_{\epsilon h} d x\right)^{2}} \frac{d}{d \epsilon}\right|_{\epsilon=0} \int_{\Omega_{\epsilon h}} u_{\epsilon h} d x=-\frac{16 V_{0}}{\pi^{2} r_{e}^{8}} \frac{\pi r_{e}^{4}}{2} \hat{h}_{0}=-\frac{8 V_{0}}{\pi r_{e}^{4}} \hat{h}_{0} .
$$

This concludes the computation of the linearization which is summarized in the next theorem.

Theorem 4.3. For $h \in h^{2+\alpha}\left(\mathbb{S}_{r_{e}}\right)$, it holds that

$$
\left.\frac{d}{d \epsilon}\right|_{\epsilon=0} G(\epsilon h)=-F^{\prime}(1)\left(\frac{4 V_{0}}{\pi r_{e}^{4}}\left[r_{e} D T N_{\mathbb{S}_{r_{e}}}(h)-h\right]+\frac{8 V_{0}}{\pi r_{e}^{4}} \hat{h}_{0}\right)
$$

Proof. The calculations preceding the formulation of the theorem yield a complete proof by observing that (4.1) implies that

$$
\left.\frac{d}{d \epsilon}\right|_{\epsilon=0} G(\epsilon h)=-F^{\prime}(1)\left[\left.\frac{d}{d \epsilon}\right|_{\epsilon=0} \lambda(\epsilon h) \partial_{\nu_{e}} \bar{u}_{e}+\left.\lambda(0) \frac{d}{d \epsilon}\right|_{\epsilon=0} \partial_{\nu_{\epsilon h}} \bar{u}_{\epsilon h}\right]
$$

and also that $\partial_{\nu_{e}} \bar{u}_{e} \equiv-1$.

Exploiting an alternative representation for the solution $w_{h}$ of

$$
\begin{cases}-\Delta w=0, & \text { in } \mathbb{B}\left(0, r_{e}\right) \\ w=h, & \text { on } \mathbb{S}_{r_{e}}\end{cases}
$$


given by

$$
w_{g}=\frac{1}{2 \pi} \hat{g}_{0}+\sqrt{2} \sum_{k=1}^{\infty} \frac{r^{k}}{r_{e}^{k}}\left[\hat{h}_{k}^{c} \cos (k \theta)+\hat{h}_{k}^{s} \sin (k \theta)\right]
$$

it is arrived at

$$
D T N_{\mathbb{S}_{r_{e}}}(h)=\partial_{\nu_{e}} w_{h}=\frac{\sqrt{2}}{r_{e}} \sum_{k=1}^{\infty} k\left[\hat{h}_{k}^{c} \cos (k \theta)+\hat{h}_{k}^{s} \sin (k \theta)\right],
$$

where $\hat{h}_{k}^{c}, \hat{h}_{k}^{s}$ are the Fourier coefficients of the function $h$ with respect to the orthonormal basis

$$
\frac{1}{2 \pi}, \sqrt{2} \cos (\theta), \sqrt{2} \sin (\theta), \sqrt{2} \cos (2 \theta), \sqrt{2} \sin (2 \theta), \ldots
$$

of $\mathrm{L}^{2}\left(\mathbb{S}_{r_{e}}\right)$. Together with representation (4.3) this yields

Theorem 4.4. The spectrum of the linearization is given by

$$
\sigma(D G(0))=-F^{\prime}(1) \frac{4 V_{0}}{\pi r_{e}^{2}}\{0,1,2,3, \ldots\} .
$$

The kernel is precisely the two-dimensional space generated by $\cos (\theta)$ and $\sin (\theta)$ due to the translation invariance of the problem as observed in Remarks 3.2 (c) and (d). The first negative eigenvalue has eigenspace generated by the functions $1, \cos (2 \theta), \sin (2 \theta)$, whereas the remaining negative eigenvalues corresponding to $k=2,3, \ldots$ have eigenspace generated by $\cos ((k+1) \theta), \sin ((k+1) \theta)$. With this knowledge of the linearization in hand, it would be possible to apply either a general center-manifold reduction approach 12 or the generalized principle of stability proved in 9]. It is arguable that more insight is, however, gained by the direct approach taken in the next section.

\section{Stability Analysis}

For the purpose of analyzing the stability of equilibria it is more convenient to use a slightly different parametrization of the manifold of curves about a fixed steady-state. Any small enough $\rho \in \mathcal{V}$ can be described using the coordinates

$$
\left(v_{1}(\rho), v_{2}(\rho), \bar{\rho}(\rho)\right)
$$

where $v=\left(v_{1}, v_{2}\right)$ is the spatial location coordinate and $\bar{\rho}$ is the "shape coordinate". In other words

$$
\left(r_{e}+\rho(\theta)\right)\left[\begin{array}{l}
\cos (\theta) \\
\sin (\theta)
\end{array}\right]=\left[\begin{array}{l}
v_{1} \\
v_{2}
\end{array}\right]+\left(r_{e}+\bar{\rho}(\theta)\right)\left[\begin{array}{l}
\cos (\theta) \\
\sin (\theta)
\end{array}\right]
$$

where $v$ is chosen so that $\bar{\rho} \in N(D G(0))^{\perp}$. The main reason for the use of this coordinate system is the fact that

Lemma 5.1. It holds that $F(\rho)=F(\bar{\rho})$.

Proof. Given $\rho \in \mathcal{V}$ consider the domains $\Omega_{\rho}$ and $v+\Omega_{\rho}$ for $v \in \mathbb{R}^{2}$. The solution $u_{v}$ of

$$
\begin{cases}-\Delta u_{v}=\lambda & \text { in } v+\Omega_{\rho}, \\ u_{v}=0 & \text { on } \Gamma_{\rho}, \\ \int_{\Omega_{\rho}} u_{v}(x) d x=V_{0}, & \end{cases}
$$

clearly satisfies

$$
u_{v}(x)=u_{0}(x-v), x \in \rho+\Omega
$$

so that

If $\theta$ is the angle variable, then

$$
\partial_{\nu_{v+\Gamma \rho}} u_{v}=\partial_{\nu_{\Gamma_{0}}} u_{0}(\cdot-v)
$$

$$
\partial_{\nu_{v+\Gamma_{\rho}}} u_{v}(\theta)=\partial_{\nu_{\Gamma_{0}}} u_{0}(\theta)
$$


and so $F(\rho)=F(v, \bar{\rho})=F(\bar{\rho})$.

In these coordinates

while

$$
V_{\rho}=\left(\dot{v}+\dot{\bar{\rho}}(\theta)\left[\begin{array}{c}
\cos (\theta) \\
\sin (\theta)
\end{array}\right] \mid \nu_{\rho}\right)
$$

It follows that

$$
\nu_{\rho}=\frac{1}{\sqrt{\left(r_{e}+\bar{\rho}\right)^{2}+\bar{\rho}^{\prime 2}}}\left[\left(r_{e}+\bar{\rho}\right) \nu_{e}-\rho^{\prime} \tau_{e}\right] \text {. }
$$

$$
\left[1+\left(\frac{\bar{\rho}^{\prime}}{r_{e}+\bar{\rho}}\right)^{2}\right]^{1 / 2} V_{\rho}=\dot{v}_{1}\left[\cos (\theta)-\frac{\bar{\rho}^{\prime}}{r_{e}+\bar{\rho}} \sin (\theta)\right]+\dot{v}_{2}\left[\sin (\theta)+\frac{\bar{\rho}^{\prime}}{r_{e}+\bar{\rho}} \cos (\theta)\right]+\dot{\bar{\rho}}
$$

Now, denoting by $\pi_{1}^{c}, \pi_{1}^{s}$, and $\pi_{1}^{\perp}$ the (orthogonal) projections onto $\mathbb{R} \cos (\theta), \mathbb{R} \sin (\theta)$, and the orthogonal complement of $\mathbb{R} \cos (\theta) \oplus \mathbb{R} \sin (\theta)$, respectively, (5.1) entails that

$$
\left\{\begin{array}{l}
\dot{v}_{1}\left\{1-\sqrt{2} \int_{0}^{2 \pi} \frac{\bar{\rho}^{\prime}}{r_{e}+\bar{\rho}} \sin (\theta) \cos (\theta) d \theta\right\}+\dot{v}_{2}\left\{\sqrt{2} \int_{0}^{2 \pi} \frac{\bar{\rho}^{\prime}}{r_{e}+\bar{\rho}} \cos ^{2}(\theta) d \theta\right\}=\pi_{1}^{c} G(\bar{\rho}), \\
\dot{v}_{1}\left\{\sqrt{2} \int_{0}^{2 \pi} \frac{\bar{\rho}^{\prime}}{r_{e}+\bar{\rho}} \sin ^{2}(\theta) d \theta\right\}+\dot{v}_{2}\left\{1+\sqrt{2} \int_{0}^{2 \pi} \frac{\bar{\rho}^{\prime}}{r_{e}+\bar{\rho}} \cos (\theta) \sin (\theta) d \theta\right\}=\pi_{1}^{s} G(\bar{\rho}), \\
\dot{\bar{\rho}}=\pi_{1}^{\perp} G(\bar{\rho})+\dot{v}_{1} \pi_{1}^{\perp}\left(\frac{\bar{\rho}^{\prime}}{r_{e}+\bar{\rho}} \sin (\theta)\right)-\dot{v}_{2} \pi_{1}^{\perp}\left(\frac{\bar{\rho}^{\prime}}{r_{e}+\bar{\rho}} \cos (\theta)\right) .
\end{array}\right.
$$

Defining the matrix $M(\bar{\rho})$ by

$$
\begin{array}{r}
M(\bar{\rho})=\left[\begin{array}{ll}
1 & 0 \\
0 & 1
\end{array}\right]+\left[\begin{array}{cr}
-\sqrt{2} \int_{0}^{2 \pi} \frac{\bar{\rho}^{\prime}}{r_{e}+\overline{\bar{\rho}}} \sin (\theta) \cos (\theta) d \theta & \sqrt{2} \int_{0}^{2 \pi} \frac{\bar{\rho}^{\prime}}{r_{e}+\bar{\rho}} \cos ^{2}(\theta) d \theta \\
\sqrt{2} \int_{0}^{2 \pi} \frac{\bar{\rho}^{\prime}}{r_{e}+\bar{\rho}} \sin ^{2}(\theta) d \theta & \sqrt{2} \int_{0}^{2 \pi} \frac{\bar{\rho}^{\prime}}{r_{e}+\bar{\rho}} \cos (\theta) \\
\sin (\theta) d \theta
\end{array}\right] \\
=\left[\begin{array}{ll}
1 & 0 \\
0 & 1
\end{array}\right]+O\left(\|\bar{\rho}\|_{h_{p}^{1+\alpha}}\right)
\end{array}
$$

and observing that it is invertible for small $\bar{\rho}$, the system reads

$$
\left\{\begin{array}{l}
\dot{v}=M(\bar{\rho})^{-1}\left[\begin{array}{c}
\pi_{1}^{c} G(\bar{\rho}) \\
\pi_{1}^{s} G(\bar{\rho})
\end{array}\right] \\
\dot{\bar{\rho}}=\pi_{1}^{\perp} G(\bar{\rho})-\pi_{1}^{\perp}\left\{\frac{\bar{\rho}^{\prime}}{r_{e}+\bar{\rho}} \tau_{e} \cdot M(\bar{\rho})^{-1}\left[\begin{array}{c}
\pi_{1}^{c} G(\bar{\rho}) \\
\pi_{1}^{s} G(\bar{\rho})
\end{array}\right]\right\}=\pi_{1}^{\perp} \widetilde{G}(\bar{\rho})=\pi_{1}^{\perp} G(\bar{\rho})+O\left(\|\bar{\rho}\|_{h_{p}^{1+\alpha}}^{2}\right) .
\end{array}\right.
$$

Notice again that the vector field in (5.2) only depends on $\bar{\rho}$ and that, by construction,

$$
\begin{aligned}
\pi_{1}^{\perp} D \widetilde{G}(0) h=\pi_{1}^{\perp} D G(0) h=-F^{\prime}(1) \frac{4 V_{0}}{\pi r_{e}^{4}}\left(\hat{h}_{0}+\sum_{k \geq 2} k\left[\hat{h}_{k}^{c} \cos (k \theta)+\hat{h}_{k}^{s} \sin (k \theta)\right]\right), & \\
\qquad & h \in \pi_{1}^{\perp} h_{p}^{2+\alpha}=: h_{p, \perp}^{2+\alpha} .
\end{aligned}
$$

It is not difficult to see that $\pi_{1}^{\perp} D G(0) \in \mathcal{H}\left(h_{p, \perp}^{2+\alpha}, h_{p, \perp}^{1+\alpha}\right)$, either by applying [2, Theorem 41] or by applying Fourier multiplier results such as those found in [1] for "periodic" symbols combined with a spectral reduction argument to split off the kernel, or by a direct computation of the associated semigroup and Fourier multiplier results.

It follows that the principle of linearized stability [8, Theorem 9.1.2] applies and yields local asymptotic stability of the trivial solution $\bar{\rho} \equiv 0$ of $\dot{\bar{\rho}}=\pi_{1}^{\perp} \widetilde{G}(\bar{\rho})$. Since the right-hand-side of (5.2) only depends on $\bar{\rho}$, since it is a smooth function of its argument, and since $G(0)=0$, it follows that

$$
v(t)=v(0)+\int_{0}^{t} M(\bar{\rho}(\tau))^{-1}\left[\begin{array}{l}
\pi_{1}^{c} G(\bar{\rho}(\tau)) \\
\pi_{1}^{s} G(\bar{\rho}(\tau))
\end{array}\right] d \tau \longrightarrow v_{\infty} \text { as } t \rightarrow \infty .
$$

The convergence is exponential since $\bar{\rho}$ converges to zero exponentially if $\bar{\rho}_{0}$ is small enough, which is always the case provided $\rho_{0}$ is. 
Theorem 5.2. Let $\left(u_{e}, \mathbb{B}\left(x_{e}, r_{e}\right)\right) \in \mathcal{E}$ be an equilibrium solution of (1.1). Then, for $\Gamma_{0}$ close enough to $\mathbb{S}_{r_{e}}$, the solution $\left(u\left(\cdot, \Gamma_{0}\right), \Omega\left(\cdot, \Gamma_{0}\right)\right)$ exists globally and there exists

$$
\rho\left(\cdot, \Gamma_{0}\right) \in \mathrm{C}^{1}\left([0, \infty), h_{p}^{1+\alpha}\right) \cap \mathrm{C}\left([0, \infty), h_{p}^{2+\alpha}\right)
$$

with

$$
\Omega\left(t, \Gamma_{0}\right)=\Omega_{\rho\left(t, \Gamma_{0}\right)} \text { for } t \in[0, \infty),
$$

as well as $v_{\infty}=v_{\infty}\left(\Gamma_{0}\right) \in \mathbb{R}^{2}$ such that

$$
\left(v_{1}(\rho), v_{2}(\rho), \bar{\rho}(\rho)\right) \longrightarrow v_{\infty} \text { as } t \rightarrow \infty
$$

exponentially fast. In other words the manifold $\mathcal{E}$ of equilibria is locally asymptotically stable and any solution, starting close to it, converges exponentially fast to a specific $\left(u_{\infty}, \mathbb{B}\left(v_{\infty}, r_{e}\right)\right)$ which depends only on the initial condition.

Proof. The details of the argument are given in the discussion preceding the theorem for a single fixed equilibrium. The same local analysis is, however, valid about any other steady-state due to the translation invariance of the problem.

It only remains to verify that $\left(v_{1}, v_{2}, \bar{\rho}\right)$ indeed provide a well-defined coordinate system for $h^{2+\alpha}\left(\mathbb{S}_{r_{e}}\right)$ about $\rho \equiv 0$.

Proposition 5.3. For any given $\rho \in \mathcal{V}$ small enough, there is a unique small $v \in \mathbb{R}^{2}$ such that

$$
\Gamma_{\rho}=\left[\begin{array}{l}
v_{1} \\
v_{2}
\end{array}\right]+\Gamma_{\bar{\rho}}
$$

for $\bar{\rho} \in N(D G(0))^{\perp}$.

Proof. Given $\rho \in \mathcal{V}$ small enough, i.e. such that $\Gamma_{\rho} \sim \mathbb{S}_{r_{e}}$, a function $\bar{\rho} \in N(D G(0))^{\perp}$ needs to be found such that

$$
\left[r_{e}+\rho(\theta)\right]\left[\begin{array}{l}
\cos (\theta) \\
\sin (\theta)
\end{array}\right]=\left[r_{e}+\bar{\rho}(\varphi)\right]\left[\begin{array}{l}
\cos (\varphi) \\
\sin (\varphi)
\end{array}\right]+\left[\begin{array}{l}
v_{1} \\
v_{2}
\end{array}\right]
$$

This identity implies that

$$
\begin{aligned}
\bar{\rho}(\varphi) & =-r_{e}+\left[\left(r_{e}+\rho\right)^{2}+|v|^{2}-2\left(r_{e}+\rho\right) v \cdot \nu_{e}\right]^{1 / 2}, \\
\tan (\varphi) & =\frac{\left(r_{e}+\rho\right) \sin (\theta)-v_{2}}{\left(r_{e}+\rho\right) \cos (\theta)-v_{1}} \\
\cot (\varphi) & =\frac{\left(r_{e}+\rho\right) \cos (\theta)-v_{1}}{\left(r_{e}+\rho\right) \sin (\theta)-v_{2}}
\end{aligned}
$$

where $\varphi=\varphi(\theta)$, and the freedom of choice between representations (5.4) and (5.5) will be exploited below. It is also useful to have

$$
\begin{aligned}
& \left(1+\tan ^{2} \varphi\right) \varphi^{\prime}(\theta)=\frac{\rho^{\prime} v \cdot \nu_{e}+\left(r_{e}+\rho\right)^{2}-\left(r_{e}+\rho\right) v \cdot \tau_{e}}{\left(\left(r_{e}+\rho\right) \cos (\theta)-v_{1}\right)^{2}}, \\
& \left(1+\cot ^{2} \varphi\right) \varphi^{\prime}(\theta)=\frac{\rho^{\prime} v \cdot \tau_{e}+\left(r_{e}+\rho\right)^{2}-\left(r_{e}+\rho\right) v \cdot \nu_{e}}{\left(\left(r_{e}+\rho\right) \sin (\theta)-v_{2}\right)^{2}} .
\end{aligned}
$$

At this point only $v$ needs to be determined. This is done by requiring that the following orthogonality conditions be satisfied

$$
\begin{aligned}
\Phi_{g}\left(v_{1}, v_{2}\right)=\int_{0}^{2 \pi} \bar{\rho}(\varphi) g(\varphi) d \varphi=\int_{\varphi(0)}^{\varphi(2 \pi)} \bar{\rho}(\varphi) g(\varphi) d \varphi \\
\quad=\int_{0}^{2 \pi} \bar{\rho}(\varphi(\theta)) g(\varphi(\theta)) \varphi^{\prime}(\theta) d \theta=0 \text { for } g=\text { sin, } \cos .
\end{aligned}
$$


Next the Jacobian of $\Phi=\left[\begin{array}{l}\Phi_{\cos } \\ \Phi_{\sin }\end{array}\right]$ is computed and is shown to be non-singular for $\delta<<1$, which then implies the claim and concludes the proof. In order to compute $\partial_{v_{1}} \Phi$, representations (5.5) /5.7) turn out to be more convenient and leads to

$$
\begin{gathered}
\partial_{v_{1}} \Phi_{g}(0,0)=-\int_{0}^{2 \pi} \frac{1}{2} \frac{2\left(r_{e}+\rho\right) \cos (\theta)}{r_{e}+\rho} \frac{\left(r_{e}+\rho\right)^{2}}{\left(1+\cot ^{2}(\theta)\right)\left(r_{e}+\rho\right)^{2} \sin ^{2}(\theta)} g(\theta) d \theta+ \\
\int_{0}^{2 \pi} \rho \frac{\left(r_{e}+\rho\right)^{2}}{\left(1+\cot ^{2}(\theta)\right)^{2}} \frac{2 \cos (\theta)}{\left(r_{e}+\rho\right)^{2} \sin ^{3}(\theta)} \frac{1}{\left(r_{e}+\rho\right) \sin (\theta)} g(\theta) d \theta+ \\
\quad-\int_{0}^{2 \pi} \rho \frac{1}{1+\cot ^{2}(\theta)} \frac{\rho^{\prime} \sin (\theta)+\left(r_{e}+\rho\right) \cos (\theta)}{\left(r_{e}+\rho\right)^{2} \sin ^{2}(\theta)} g(\theta) d \theta \\
\quad+\int_{0}^{2 \pi} \rho \frac{\left(r_{e}+\rho\right)^{2}}{1+\cot ^{2}(\theta)} \frac{1}{\left(r_{e}+\rho\right)^{2} \sin ^{2}(\theta)}\left(\left.\partial_{v_{1}}\right|_{(0,0)} g(\varphi)\right) d \theta \\
=\int_{0}^{2 \pi}\left\{-\cos (\theta)+2 \frac{\rho}{r_{e}+\rho} \cos (\theta)-\frac{\rho}{\left(r_{e}+\rho\right)^{2}}\left[\rho^{\prime} \sin (\theta)+\left(r_{e}+\rho\right) \cos (\theta)\right]\right\} g(\theta) d \theta+\int_{0}^{2 \pi} \rho(\theta) g^{\prime}(\theta) d \theta
\end{gathered}
$$

where the last term is obtained by observing that

$$
\left.\partial_{v_{1}}\right|_{(0,0)} g(\varphi)=\left.g^{\prime}(\varphi) \varphi^{\prime}\right|_{(0,0)}=g^{\prime}(\theta) \frac{\left(r_{e}+\rho\right)^{2}}{\left(1+\cot ^{2}(\theta)\right)\left(r_{e}+\rho\right)^{2} \sin ^{2}(\theta)}=g^{\prime}(\theta), \theta \in[0,2 \pi) .
$$

It follows that

$$
\begin{array}{ll}
\partial_{v_{1}} \Phi_{\cos }(0,0)=-1 / 2+O\left(\|\rho\|_{h_{p}^{1+\alpha}}\right) & \text { as } \rho \rightarrow 0, \\
\partial_{v_{1}} \Phi_{\cos }(0,0)=O\left(\|\rho\|_{h_{p}^{1+\alpha}}\right) & \text { as } \rho \rightarrow 0 .
\end{array}
$$

In an analogous manner, but using representations (5.4)/(5.6) instead, it can be seen that

$$
\begin{aligned}
& \partial_{v_{2}} \Phi_{\cos }(0,0)=O\left(\|\rho\|_{h_{p}^{1+\alpha}}\right) \quad \text { as } \rho \rightarrow 0, \\
& \partial_{v_{2}} \Phi_{\sin }(0,0)=-1 / 2+O\left(\|\rho\|_{h_{p}^{1+\alpha}}\right) \text { as } \rho \rightarrow 0,
\end{aligned}
$$

so that the proof is complete.

Remark 5.4. The reduction performed in this paper is similar to that required in the proof of the generalized principle of stability [9] already mentioned. Two essential differences need, however, to be pointed out. On the one hand, the generalized principle of linearized stability applies in general to abstract problems exhibiting the right structure as identified in [9], while the use of nonlinear coordinates in the space of shapes chosen here is responsible for a simpler normal form and, thus, a much simpler proof. Since the geometric nonlinear coordinates are tangent, at the steady-state, to the linear ones in which the linearization is computed, the relevant linearized operator is actually the same.

\section{REFERENCES}

[1] W. Arendt and S. Bu. Operator-valued fourier multipliers on periodic besov spaces and applications. Proc. Edinb. Math. Soc., 47:15-33, 2004.

[2] J. Escher and P. Guidotti. Local Well-posedness for a quasi-stationary droplet model. submitted.

[3] J. Escher and G. Simonett. The volume preserving mean curvature flow near spheres. Proceedings of the american Mathematical Society, 126(9):2789-2796, 1998.

[4] K. B. Glasner. A boundary integral formulation of quasi-steady fluid wetting. Phys., 207:529-541, 2005.

[5] H. P. Greenspan. On the motion of a small viscous droplet that wets a surface. J. Fluid Mech., 84:125-143, 1978.

[6] I. Kim and C.K. Glasner. Viscosity solutions for a model of contact line motion. Interfaces Free Bound., 311(1):37-60, 2009. 
[7] I. Kim and N. Grunewald. A variational approach to a quasi-static droplet model. Calc. Var. Partial Differential Equations, 41(1-2):1-19, 2011.

[8] A. Lunardi. Analytic Semigroups and Optimal Regularity in Parabolic Problems. Birkhäuser, Basel, 1995.

[9] J. Prüss, G. Simonett, and R. Zacher. On convergence of solutions to equilibria for quasilinear parabolic problems. Journal of Differential Equations, 246(10):3902-3931, 2009.

[10] E. Schippers. Behaviour of kernel functions under homotopic variations of planar domains. Computational Methods and Function Theory, 4(2):283-298, 2005.

[11] J. Serrin. A symmetry problem in potential theory. Archive for Rational Mechanics and Analysis, 43(4):304$318,1971$.

[12] G. Simonett. Center manifolds for quasilinear reaction-diffusion systems. Differential and Integral Equations, 8(4):753-796, 1995.

University of California, Irvine, Department of Mathematics, 340 Rowland Hall, Irvine, Ca 926973875 , USA

E-mail address: gpatrick@math.uci.edu 